\title{
Childbirth and risks to maternal health in the obstetric culture of the Siendji Brong in Côte d'lvoire
}

\begin{abstract}
This paper examines the typology of childbirth of the Siendji Brong in Côte d'Ivoire, and highlights the associated psychosocial perceptions and medical practices that endanger maternal health. The research was qualitative, with an ethnographic scope. Data was collected from two focus groups, women over 50 and multiparas respectively, in order to identify the community's physiological criteria for classifying childbirth. Semi-structured interviews were also conducted with traditional midwives and parturients' caregivers regarding psychosocial and medical aspects of childbirth. Through content analysis, informants' representations regarding childbirth and the mechanisms underlying the risks to maternal health were brought out. The community distinguished primarily between kou gariogo, "childbirth with complications", and kou tchrêssêguê, "uncomplicated childbirth". Risks to maternal health stemmed from ignorance of warning signs and the practice of home birth, both perpetuated by local obstetrical culture. Communication and education are needed for social change that would reduce reproductive health risks in the community.
\end{abstract}

Keywords: high-risk, maternal health, obstetric culture, parturition, siendji brong
Volume 6 Issue 2 - 202I

\author{
Adou Serge Judicaël Anoua \\ Department of Anthropology \& Sociology, Research and Training \\ Unit Communication, Milieu and Society, Alassane Ouattara \\ University, Bouaké, Côte d'Ivoire
}

\begin{abstract}
Correspondence: Department of Anthropology \& Sociology, Research and Training Unit Communication, Milieu and Society, Alassane Ouattara University, Bouaké, Côte d'Ivoire, Tel+2250707070136,Email anoua_08@yahoo.fr
\end{abstract}

Received:September 17, 2021 | Published: October 12, 202 |

\section{Introduction}

Childbirth, defined as the emergence of the fetus from the uterus and into the outside world, continues to present an alarmingly high risk of complications or mortality for both the parturient and the neonate. Frequent complications include lesions, infections, and disabilities. On the whole, the issue of maternal and neonatal mortality remains a pressing concern for public health worldwide. Most cases of mortality are evitable and occur in low-income countries. In 2015, 303,000 maternal deaths during pregnancy or following childbirth were recorded across the globe, but the vast majority (99\%) occurred in developing regions, and Sub-Saharan Africa alone accounted for 66 percent of this mortality rate. ${ }^{1}$ In 80 percent of cases, maternal mortality is essentially attributable to direct medical causes such as severe haemorrhage, postpartum infections, other complications of childbirth, abortion performed under unsafe conditions, preeclampsia, and eclampsia. ${ }^{2}$ These various causes of maternal mortality have given rise to numerous actions aimed at lowering its rate worldwide. A notable example is the acceleration framework for Millennium Development Goal 5 (MDG 5), with its three main intervention areas, namely family planning, access to emergency obstetric and neonatal care, and monitoring pregnancies and the health of mothers and new-borns in the postpartum period. ${ }^{3}$ In the same spirit, efforts to improve maternal health have been made within the framework of the Sustainable Development Goals (SDG). In particular, SDG 3 represents a new initiative to transform maternal health worldwide with the ultimate goal of eradicating evitable maternal deaths. The first target of this goal is the reduction of the rate of worldwide maternal mortality to less then 70 per 100,000 live births by $2030 .^{4}$

Despite such efforts, maternal and child health remains a cause for concern in Côte d'Ivoire. According to official data, the country's maternal mortality rate for 2011-2012 was as high as 614 per 100,000 live births, whereas the corresponding rate for infants less than a year old was 63.02 per 1000 normal births. ${ }^{5}$ Furthermore, the national rate of births assisted by qualified medical professionals went from 64.6 percent in 2017 to 64.1 percent in 2018 , which amounts to a decrease of 0.77 percent (MSHP, 2018). This deterioration raises the question of why unassisted births still persist in the present day, despite awareness campaigns and other measures taken to reduce maternal mortality. Some answers can be gleaned from the literature; for instance, ${ }^{6}$ have noted lack of education, lack of prepartum consultations, limited financial means, and high costs associated with an assisted birth as determinants which predispose expectant mothers to a home birth. Kané ${ }^{7}$ in turn, lists low accessibility of healthcare facilities, high parity, and insufficient information, education and communication as factors relevant to a preference for home births. Moreover, cultural perceptions can play a role in deterring expectant mothers from an assisted birth. A case in point are the nomadic women in Gossi, Mali, who consider that childbirth is a natural phenomenon and consequently requires no medical intervention, and whose ability to make autonomous decisions is in any case limited. They are also anxious about being undressed, giving birth on a table, and above all, being seen by male medical staff. ${ }^{8}$ From this perspective, the human dimension, and particularly the traditional knowledge that has evolved around childbirth in individual communities, is crucial in parturients' aversion to an assisted birth. In the region of Gontougo in Côte d'Ivoire, the 2018 rate of assisted births was intermediate at 64.1 percent, which fell below the national target of 68.8 percent for that year. ${ }^{9}$

The Siendji Brong community, who reside in the healthcare area of Siasso, are no exception to this trend in reproductive health. Their approach to childbirth is guided by social and cultural perceptions, including the dichotomy between kou gariogo, "childbirth with complications", meaning dystocia, and kou tchrêssêguê, "uncomplicated childbirth". This fundamental two-way classification of childbirth is based on an interpretation of physiological criteria which is at odds with modern medicine. Siendji Brong obstetrical culture as a whole involves practices related to reproductive health 
which favour childbirth unassisted by qualified medical professionals. As a result, expectant mothers in this rural community are exposed to serious health risks. An anthropological approach within an ethnographic perspective was chosen as the basis for research, given that an understanding of the culture of the Siendji Brong of Siasso and of their traditional gynaecological and obstetrical knowledge is necessary to account for their attitudes and practices in relation to childbirth and the risks to which the latter expose expectant mothers. This paper aims to explore the typology of childbirth as recognised by the Siendji Brong of Siasso in order to elucidate the associated psychosocial perceptions and medical practices which pose a risk to maternal health

\section{Material and methods}

The social realities of the Brong Siendji need be considered in the geographic and cultural context of Côte d'Ivoire. The autochthonous population of Côte d'Ivoire is composed of over 60 ethnicities, which can be subsumed into four broad ethnocultural groups, namely Akan, Gur, Mande and Kru. Each of these groups is distinguished by an original set of beliefs regarding procreation. This paper is dedicated to the Brong population of Eastern Côte d'Ivoire, which belongs to the Akan group. Field research was carried out in three Siendji-speaking Brong villages - Djadoubango, Siendji-Sogo and Secrebango - in the sub-prefecture of Tiédio in the department of Tanda in the region of Gontougo. The villages in question are secluded and do not have their own healthcare centres but fall within the broader healthcare area of Siasso. The latter locality has a single rural healthcare centre which women from the three villages sometimes visited for prepartum consultations and in order to give birth. Research was conducted over a period of two weeks, between the $2^{\text {nd }}$ and the $15^{\text {th }}$ of April 2021, with a qualitative approach and within an ethnographic scope. Interviews with women aged over 50, multiparas, traditional midwives and parturients' caregivers shed light on these social groups' understanding of childbirth, within the ethnocultural context of the Siendji Brong community. Based on the gathered data, the typology of childbirth according to the traditional gynaecological and obstetrical knowledge of the Siendji Brong, as well as the associated psychosocial perceptions and medical practices which pose a risk to maternal health, were identified and assessed against modern epidemiological concepts.

In compliance with ethical standards, participants were guaranteed anonymity, confidentiality, and the right to withdraw from the study at any time, and each of them gave informed consent after having been acquainted with the research objectives and methods. The methods of data collection were focus groups and semi-structured interviews, which were rigorously carried out in the three villages. In each village, two focus groups were conducted with two target populations, namely women aged over 50 and multiparas who had a child less than 5 years old and who had delivered at home at least once. The first target population was chosen because of their greater experience with procreation, including that which they had indirectly acquired as grandmothers or mothers-in-law involved in the reproductive lives of their daughters or daughters-in-law respectively. The second target population was similarly chosen because they had experienced motherhood multiple times, as well as home births, in keeping with the local culture under study. The semi-structured interviews, in turn, were carried out with traditional midwives and parturients' caregivers, who had experience with offering psychosocial and medical care on the occasion of childbirth and in the post-partum period. Table 1 summarises the statistical data regarding the totality of the participants.

Table I Study population

\begin{tabular}{lllll}
\hline & Focus group & \multicolumn{2}{c}{ Semi-structured interview } \\
\hline Villages & Women aged over $\mathbf{5 0}$ & Multiparas & Traditional midwives & Parturients' caregivers \\
\hline Djadoubango & 10 & 12 & $\mathrm{I}$ & 0 \\
Siendji-Sogo & 11 & 12 & $\mathrm{I}$ & $\mathrm{I}$ \\
Secrebango & 8 & 7 & $\mathrm{I}$ & 0 \\
Total & 29 & 31 & 3 & 1 \\
\hline
\end{tabular}

Source: field research in the healthcare area of Siasso,April 2021

The gathered data was manually sorted and processed, based on procedures described in Miles et al. ${ }^{10}$ Specifically, the verbal data was represented as complete sentences, so that each could be better analysed in context and so that the significant segments could be brought to the fore. Through a comprehensive consideration of the stances expressed by the interviewed women, a synthetic inventory of their specific ideas was put together and the main themes which underly them were identified. By means of content analysis, ${ }^{11}$ the data was categorised into perceptions, explanations, and social determinants, in order to account for the attitudes and approach to childbirth of the interviewed women. Once a structured view of the relevant information had been obtained, the practices related to childbirth which are harmful to the quality of mothers' reproductive lives were singled out.

\section{Results}

The Siendji Brong refers to childbirth as koum gbô. They are aware that the phenomenon is a natural part of reproduction and that complications related to physiological factors can occur. They distinguish two types of childbirth, namely kou gariogo, "childbirth with complications", and kou tchrêssêguê, "uncomplicated childbirth", which can be understood as non-dystocic labour with low risks.

\section{Kou gariogo, or childbirth with complications}

According to the Siendji Brong, this type of childbirth involves one of two risk factors: a transverse lie of the fetus in the uterus, referred to in the local vernacular as bi braca, or obstruction of the uterus, known as zouko.

\section{Kou gariogo due to Bi Braca, or obstructed labour due to a transverse lie of the fetus}

This type of childbirth involves complications due to a transverse lie of the fetus. Because of this malpresentation, childbirth can last for hours, as the traditional midwives stated. Thus, labour is long and difficult and the parturient and the fetus are at a higher risk of death. According to the midwives, a warning sign of this type of obstructed 
labour can be the lack of lightening prior to childbirth, that is to say, if the baby fails to settle into the pelvis before being delivered. This is considered abnormal and is designated by the term foum'gbo djiran, or blocked womb. The perceived association between a lack of lightening and difficult childbirth can be illustrated with the following testimony from a midwife from Siendji-Sogo: Not everybody is aware of this, but when the mother's baby is due, it should drop first. However, there are some pregnant women whose baby bump doesn't lower before they go into labour. When I assist a woman in that situation, I tell her relatives to take her to a healthcare centre if she is in any pain, because the childbirth is going to be a difficult one. In the past, we would take the risk, but now, things have changed. (Traditional midwife from Siendji-Sogo).

Parturients' caregivers emphasised that in order to forestall difficult labour, it is necessary to provide medicinal treatment to the expectant mother during pregnancy and delivery, as one caregiver from SiendjiSogo explained:

Women often come to us so that we can help them. The help consists of giving them medicine made from medicinal plants, so that the pregnancy and delivery can follow a normal course. This requires that the baby is properly positioned in the womb. That way, delivery will be easier. Those who know me come to me and I do what I can. If they end up giving birth without complications, they come back to thank me. (Caregiver from Siendji-Sogo). Now that the transverse lie of the fetus has been examined, a discussion of the obstruction of the uterus is in order.

\section{Kou gariogo due to zouko, or obstructed labour due to obstruction of the uterus}

This type of childbirth involves complications due to an alleged obstruction of the uterus by a membrane at the time of childbirth, a phenomenon locally referred to as zouko. In this case, it is considered that a physical barrier prevents the fetus from emerging from the uterus and thus protracts labour. The women aged over 50, multiparas and traditional midwives who were interviewed regarded the phenomenon as posing a serious risk. According to them, it could lead to the deaths of both mother and infant, or alternatively handicap the mother, who might limp due to pain in her leg, explained as a result of the fetus penetrating into her thigh. The vernacular term for this complication is bi san houkôn, described as follows by one of the women aged over 50 from Djadoubango: We are very afraid of this type of childbirth. There have been cases around here, but very long ago. Delivery lasted for days. Afterwards, the woman did not have any more children. She couldn't, in fact, because she developed a limp. It was said that the baby had gone into her thigh. The woman is still alive. Everyone knows that she has never been able to recover from her difficult childbirth. In any case, women feel more comfortable with traditional midwives when they give birth, because they are experienced. (Focus group with women aged over 50 in Djadoubango). In addition to these two types of obstructed labour, the participants also spoke of normal childbirth with low risks.

\section{Kou Tchrêssêguê, or uncomplicated childbirth}

The second main type of childbirth recognised by the Siendji Brong is kou tchrêssêguê, understood as normal childbirth with low risks. The traditional midwives who were interviewed claimed that any difficulties that arise during this type of childbirth are easily dealt with and successfully resolved. The complications that participants assigned to this category include postpartum haemorrhage, or tohom sohôko, and a retained placenta, or djiha hon.

\section{Kou Tchrêssêguê accompanied by Tohom Sohôkô, or non-dystocic labour accompanied by postpartum haemorrhage}

In Siendji Brong culture, when childbirth is followed by postpartum haemorrhage, or tohom sohôkô, traditional midwives provide speedy treatment. As the interviewed multiparas who had already had a home birth revealed, most of the midwives who had tended to them had recipes for treating the blood loss. Nevertheless, postpartum haemorrhage is by no means risk-free, since the midwives do not always succeed in stopping it, as the multiparas interviewed at Djadoubango related: What sets traditional midwives apart is that some of them have recipes for stopping the bleeding after delivery. When I gave birth to my first child, I was bleeding and the midwife who assisted me had difficulty in stopping it. Otherwise, childbirth offers no surprises for traditional midwives. Also, we don't choose to deliver at home; circumstances compel us, and then we always have recourse to midwives. After my first experience with a midwife, I delivered my other children in a maternity ward. (Focus group with multiparas in Djadoubango).

It should be pointed out that every traditional midwife has her own medicinal products which differentiate her. The participants noted that the medicine administered to a parturient during childbirth includes kaolinite and infusions made with medicinal plants, the precise nature of the ingredients being jealously guarded. A traditional midwife from Secrebango described her services in the following terms: I have helped several women deliver their child and I continue to help them as long as they ask me to. The last time I assisted childbirth was about two months ago. It was after noon and my neighbour called me because her daughter was going into labour. It was impossible to drive her to the healthcare centre in Tiédio in her condition. Without hesitating, I assisted her myself. She bled after delivery, but I'm used to that, so I gave her what she needed and the bleeding stopped. I gave her medicinal kaolinite, which I had gotten from my mother. I rub it in a gourd filled with water and I let the woman who's bleeding drink from it. (Traditional midwife from Secrebango). Besides postpartum haemorrhage, the Siendji Brong also point to a retained placenta among the complications accompanying what they otherwise perceive to be normal childbirth.

\section{Kou Tchrêssêguê accompanied by Djiha Hon, or non- dystocic labour accompanied by a retained placenta}

In the language of the Siendji Brong, a retained placenta is called djiha hon. This condition was not considered to be a serious complication by the women aged over 50 and the traditional midwives, as the latter believed that they always found a way to manage it when assisting parturients at a home birth. The women aged over 50 who were interviewed claimed that midwives made the placenta detach by applying kaolinite onto the stomach of a parturient. They added that a retained placenta would never lead them to seek medical attention in a healthcare centre. However, the experiences reported by the multiparas from Siendji-Sogo reveal that a retained placenta is not a negligible risk in the community, as shown by the following testimony from one of the multiparas regarding her second home birth: When I gave birth at home for a second time with the help of my mother, I almost lost my life. Everything went well during the delivery itself, but afterwards, my placenta wouldn't come out, despite an intervention by a traditional midwife from the village who arrived later. I ended up in the healthcare centre of Tiédio. Without the assistance of the midwife, perhaps I wouldn't be here today. Otherwise, none of the other women here who have given birth at home have experienced 
any of the difficulties that I underwent. (Focus group with multiparas in Siendji-Sogo).

From these reports about childbirth, it emerges that home births are embedded in the culture and values of the Siendji Brong in the healthcare area of Siasso. The preference for home births has consequences for the quality of mothers' reproductive lives in this ethnocultural group. These findings will be further clarified in the following section.

\section{Discussion}

From a biomedical point of view, the perceptions and practices relating to childbirth in the obstetric culture of the Siendji Brong pose risks to maternal health.

\section{Risks posed by the obstetric culture of the Siendji Brong}

In the obstetric culture of the Siendji Brong, two main types of childbirth are distinguished, kou gariogo, "childbirth with complications", and kou tchrêssêguê, "uncomplicated childbirth". This opposition provides insight into the perception of childbirth in the ethnocultural context under study and the underlying social representations. The concept of kou gariogo refers to childbirth which entails serious risks to maternal health, due to a transverse lie of the fetus, bi braca, or obstruction of the uterus, zouko. For the Siendji Brong, these two complications are the only ones which cause childbirth to be perceived as abnormal and problematic. Since such obstructed labour imperils the health of the mother and the fetus, when it presents during a home birth, traditional midwives advise recourse to a healthcare centre, where the dystocia can be managed by qualified medical professionals. Pourette ${ }^{12}$ notes that in Madagascar too, traditional midwives acknowledge their inability to manage certain pathologies and complications associated with pregnancy and childbirth and that they consequently raise no objection to the services provided by medical professionals; they do not call biomedical treatments into question and content themselves with offering auxiliary care.

As for kou tchrêssêguê, it is understood as normal childbirth which poses low risks to maternal health, but which can still be accompanied by postpartum haemorrhage, tohom sohôkô, or a retained placenta, djiha hon. In such cases, the Siendji Brong believe that the situation is under control and that the complications can be successfully treated with kaolinite and medicinal plants. Yet postpartum haemorrhage remains the leading cause of maternal mortality. This trend has also been observed at a local level by Mbeva et al., ${ }^{13}$ whose study found postpartum haemorrhage to be the leading cause of maternal mortality in hospital conditions in the east of the Democratic Republic of the Congo. Thus, it is evident that the complications considered to be less severe by the Siendji Brong in fact entail a significant risk of maternal death. The literature can shed light on the reasons for which such complications are not taken seriously enough or dealt with adequately. For instance, Kouamé et al., ${ }^{14}$ have pointed out that in Côte d'Ivoire, ignorance of the warning signs of postpartum haemorrhage is a major factor responsible for delays in deciding to seek out emergency obstetric care and that tenacious social perceptions deter populations from doing so. Furthermore, Sepou et al. ${ }^{15}$ remark in their study on maternal mortality in the Central African Republic that the period of pregnancy itself is often enshrouded in prejudice and that certain cultural constraints restrict access to emergency obstetric care. Zaouaq $^{16}$ in turn, argues in his study on access to obstetric care in Morocco that low levels of education among women and their lack of awareness about their reproductive health make them more susceptible to the influence of cultural factors when it comes to seeking out such care. It seems sensible to extend these findings to the Siendji Brong, since they are a rural African community like those studied in the cited works. Risks to maternal health stem not only from the obstetric perceptions of the Siendji Brong regarding childbirth, but also from the practice of home birth.

\section{Risks related to the practice of home birth}

Traditional midwives and caregivers tending to parturients during a home birth is a part of the obstetric culture of the Brong Siendji in the healthcare area of Siasso, as the preceding discussion has shown. Care is delivered to ease labour, but also to provide a therapeutic response to the various obstetric complications presented by parturients. Akoto et al., ${ }^{17}$ have noted that in Cameroon too, the services of traditional midwives are still regularly solicited by pregnant women. In this regard, Pourchez ${ }^{18}$ has pointed out that possessing genuine obstetric skills is crucial for traditional midwives' reputations. This is borne out in the case of the Siendji Brong, who value traditional midwives' skills and experience; consequently, they are disinclined to seek assistance by qualified medical professionals when they give birth. Moreover, home births are deeply rooted in tradition, just as Paraïso et al., ${ }^{19}$ have observed among the Holly ethnic group in the healthcare zone of Pobè-Adja-Ouèrè-Kétou, Benin. Nkurunziza ${ }^{20}$ in turn, found that in rural Burundian communities, home births remain popular despite the availability of free healthcare, due to a preference for traditional midwives, families' meagre financial means, and women's limited financial and decision-making autonomy.

The care provided by traditional midwives and caregivers in the Siendji Brong community relies on traditional African medicine, as can be seen in the use of substances such as kaolinite and medicinal plants. Such therapeutic practices can in fact prove harmful, insofar as they can give rise to infections and postpartum haemorrhage, endangering the lives of the mother and the fetus. The risks associated with childbirth unassisted by qualified medical professionals have already been discussed in the context of other African countries; for example, Kané ${ }^{7}$ found that in Commune II of Bamako, Mali, haemorrhage due to uterine atony was the most frequent complication. A second study of unassisted childbirth in Bamako by Cheickna et al. ${ }^{21}$ centred on the epidemiological aspects of the practice, found a non-negligible rate of occurrence for multiple serious complications including haemorrhage due to uterine atony, perineal tears, a retained placenta, fetal distress and stillbirths. Given this multitude of risks associated with home births, the practice is clearly harmful to the welfare of the Siendji Brong community of Siasso. In order to reduce its prevalence, it is necessary to address the factors that account for it, such as the unavailability of a maternity hospital in the village where parturients live, the high costs associated with childbirth assisted by medical professionals, the remote location of the relevant healthcare facilities, and the ill-treatment of parturients in such facilities, as suggested by Kwete et al., ${ }^{22}$ in the context of the Democratic Republic of the Congo. Remedying these problems could pave the way for a solution in the specific case of the Siendji Brong in the healthcare area of Siasso.

\section{Conclusion}

Siendji Brong obstetric culture distinguishes two types of childbirth, namely kou gariogo, "childbirth with complications", or dystocic labour with high risks, and kou tchrêssêguê, "uncomplicated childbirth", or non-dystocic labour with low risks. The traditional obstetric culture imperils maternal health. In particular, parturients 
are exposed to risk due to insufficient knowledge of warning signs of Peripartum complications in the community and the prevalent practice of home birth. Be that as it may, the sociocultural perceptions and practices of the Siendji Brong surrounding childbirth are engrained in tradition and withstand the test of time. As such, they deter parturients from opting for childbirth assisted by qualified medical professionals. Understanding the intricacies of Siendji Brong obstetric culture is necessary before working towards reshaping the community's representations of childbirth and challenging their untenable cultural convictions. Such convictions and the dangerous practices that they bolster need to be overcome in the interest of the health and survival of mothers and their children. The transformational process needs to be conducted through carefully planned interventions. Firstly, educational and communication strategies need to be conceived in order to bring out about social and behavioural change. To this end, it is indispensable to set up and develop tools and structures for informing and educating the population about childbirth and the proper practices associated with it; that way, awareness could be raised within the community. Further promotional activities and community participation would be favourable to an efficient campaign against harmful traditional practices.

In sum, educating the Siendji Brong of the healthcare area of Siasso is essential to combating high-risk behaviours related to childbirth and to short- or medium-term improvement of the quality of women's reproductive lives in this rural community in Côte d'Ivoire. Furthermore, and obviously irrational beliefs and behaviours rooted in the obstetric culture of the Siendji Brong of the healthcare area of Siasso lead to systematic resistance to childbirth assisted by qualified medical professionals. To combat this trend, the following recommendations are made:

1. Develop and reinforce the cross-cultural skills of midwives and provide them with the necessary anthropological knowledge to meet parturients' expectations in relation to childbirth.

2. Encourage traditional midwives to immerse themselves into the social lives of pregnant women and work towards reducing the rate of home births.

3. Implement modern and realistic strategies, true to biomedical principles, in order to achieve social and behavioural change through communication and education about the proper practices related to childbirth, aimed at pregnant women, their spouses, their relatives, traditional midwives, and caregivers.

4. Foster community participation in the fight against the prevalence of home births in order to ensure that the social change in progress does not come to a standstill and to combat individual resistance to change.

5. Use petitions and efficient community action to urge public authorities to get involved and work on conditions in rural areas, where access to healthcare centres and the services offered to parturients leave much room for improvement.

\section{Acknowledgments}

The author is grateful to the women aged over 50, multiparas, traditional midwives, and parturient caregivers of Brong Siendji for the many hours that they devoted to this study, and collaboration during the data collection period.

\section{Conflicts of interest}

The author declares that there is no conflict of inertest.

\section{Funding}

None.

\section{References}

1. Alkema L, Chou D, Hogan D, et al. Global, regional, and national levels and trends in maternal mortality between 1990 and 2015, with scenariobased projections to 2030: a systematic analysis by the UN maternal mortality estimation inter-agency group. Lancet. 2016;387(10017):462474.

2. Say L, Chou D, Gemmill A, et al. Global causes of maternal death: a WHO systematic analysis. The Lancet Global Health. 2014:2(6):e323e333.

3. United Nations, UN. Framework for accelerating achievement of the MDGs. New York: United Nations development group (UNDG). 2011.

4. World Health Organization, WHO. Trends in maternal mortality: 19912015. Estimates from WHO, UNICEF, UNFPA, World bank group and United Nations population division. Executive summary (No. WHO / RHR / 15.23). Geneva: World health organization. 2015.

5. National Institute of Statistics (INS) and ICF International. Demographic and health survey and multiple indicators of Côte d'Ivoire 2011-2012. Calverton, Maryland, USA: INS and ICF International. 2012.

6. Sangho O, Traoré SO, Kamate YD, et al. Comparison of the determinants of home birth in two neighborhoods in commune $\mathrm{V}$ of Bamako. Mali Medical. 2020; 35(3):63-69.

7. Kané Salimata. Factors and complications associated with unassisted deliveries in the reference health center of commune II of Bamako, in 2019. M. Phil. Dissertation. Bamako: University of sciences, techniques and technologies of Bamako. 2020:1-38pp.

8. Ahmed Mohamed Ali Ag. Determinants of whether or not childbirth is used assisted by nomadic women from Gossi in Mali and potential strategies to facilitate it. Ph. D. Thesis. Quebec: Laval University. 2019:1224pp.

9. Ministry of Health and Public Hygiene (MSHP). Annual report on the health situation 2018. Abidjan: Republic of Côte d'Ivoire. 2019.

10. Miles MB, Huberman MA. Analyse des données qualitatives. $2^{\text {nd }}$ Edition. Translated by Rispal MH. Bruxelles: De Boeck. 2003:1-626pp.

11. Paillé P, Mucchielli A. Qualitative analysis in human and social sciences. $3^{\text {rd }}$ Edition. Paris: Armand Colin. $2012: 1-424 p p$.

12. Pourette D. The “matrons" in Madagascar. Local recognition, institutional denial and collaborations with professionals. In: Dolorès Pourette, Chiarella Mattern, Christine Bellas Cabane, Bodo Ravololomanga (Eds.): Women, children and health in Madagascar. Comparative anthropological approaches. Paris: L'Harmattan.2018:115-126pp.

13. Mbeva JBK, Karemere H, Prudence MN, et al. Maternal deaths factors in hospital area: a survey at six health districts in the east of the republic democatique of Congo. International Journal of Innovation and Applied Studies. 2018;23(4):559-568.

14. Kouamé ZPG, Anoua ASJ, Abé NDN. High risk of postpartum haemorrhage at Bouaké University Hospital (North-Central Côte d'Ivoire). Anthropological Researches and Studies. 2021;1(11):78-91.

15. Sepou A, Serdouma E, Komas NP, et al. Strategies for reducing maternal mortality in the Central African republic. Strategic briefing note. Geneva: World health organization. 2011

16. Zaouaq $\mathrm{K}$. Women and access to reproductive health care in Morocco. L'Année du Maghreb. 2017;17:169-183.

17. Akoto EM, Songue PB, Lamlenn S, et al. Private nurses, traditional healers, traditional birth attendants in the countryside and in the city. Bulletin de l'APAD. 2001;21:1-14. 
18. Pourchez L. Knowledge of women. Traditional medicine and nature (Mauritius, Rodrigues, Reunion). The Health Tribunes. 2014;3(3):51-71.

19. Paraïso NM, Agueh VD, Nkwidjan H, et al. Home births in the health zone of Pobè-Adja-Ouèrè-Kétou in 2011, Benin. J Epidemiol Publ Health. 2012;60:S136.

20. Nkurunziza M. Giving birth at home despite free healthcare: The case of rural Burundi. Autrepart. 2015;2(2-3):85-100.
21. Cheickna S, Oumar TS, Saleck D, et al. Unsured deliveries in urban areas in Bamako, Mali. Health Sciences and Disease. 2020;2(12):73-78.

22. Kwete MB, Nyota RB, Mbarambara PM. Factors associated with home delivery in rural health zone of Lemera, DR Congo]. International Journal of Innovation and Applied Studies. 2016;17(4):1183-1189. 